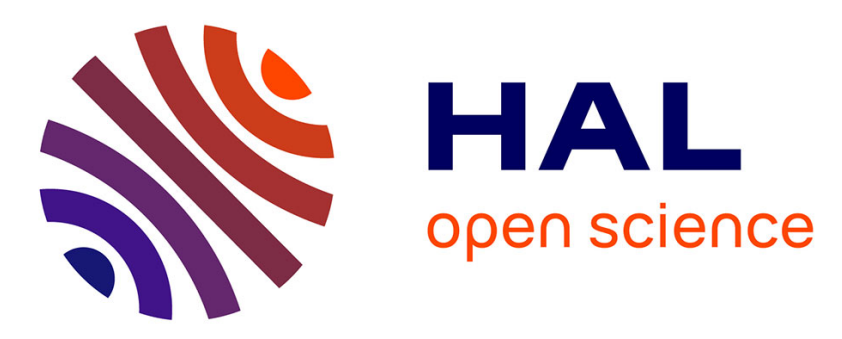

\title{
Posture and body acceleration tracking by inertial and magnetic sensing: Application in behavioral analysis of free-ranging animals
}

Hassen Fourati, Noureddine Manamanni, Lissan Afilal, Yves Handrich

\section{To cite this version:}

Hassen Fourati, Noureddine Manamanni, Lissan Afilal, Yves Handrich. Posture and body acceleration tracking by inertial and magnetic sensing: Application in behavioral analysis of free-ranging animals. Biomedical Signal Processing and Control, 2011, 6 (1), pp.94-104. 10.1016/j.bspc.2010.06.004 . hal00642357

\section{HAL Id: hal-00642357 \\ https://hal.science/hal-00642357}

Submitted on 18 Nov 2011

HAL is a multi-disciplinary open access archive for the deposit and dissemination of scientific research documents, whether they are published or not. The documents may come from teaching and research institutions in France or abroad, or from public or private research centers.
L'archive ouverte pluridisciplinaire HAL, est destinée au dépôt et à la diffusion de documents scientifiques de niveau recherche, publiés ou non, émanant des établissements d'enseignement et de recherche français ou étrangers, des laboratoires publics ou privés. 


\title{
Posture and body acceleration tracking by inertial and magnetic sensing: Application in behavioral analysis of free-ranging animals
}

\author{
Hassen Fourati ${ }^{\mathrm{a}, \mathrm{b}}$, Noureddine Manamanni ${ }^{\mathrm{a}, *}$, Lissan Afilal $^{\mathrm{a}}$, Yves Handrich ${ }^{\mathrm{b}}$ \\ ${ }^{a}$ CReSTIC, URCA, EA 3804 - Université de Reims Champagne-Ardenne \\ UFR SEN, Moulin de la Housse Bat 12, 51687 Reims Cedex 2 France \\ \{name.surname\}@univ-reims.fr \\ (*Corresponding author: Tel: +33326918386; Fax: +33326913106) \\ ${ }^{b}$ Institut Pluridisciplinaire Hubert CURIEN / Département Ecologie, Physiologie et Ethologie \\ UMR 7178 CNRS - Université de Strasbourg \\ 23 rue du loess - BP28 67037 Strasbourg cedex 2, France. \\ hassen.fourati@c-strasbourg.fr, yves-jean-handrich@c-strasbourg.fr
}

\begin{abstract}
This paper concerns body attitude (orientation) estimation for free ranging animal. The main idea of the proposed approach combines a quaternion-based nonlinear observer with an Iterated Least Squares Algorithm (ILSA) and exploits measurements from Micro-Electro-Mechanical-System (MEMS) sensors as 3-axis accelerometer, 3-axis magnetometer and 3-axis gyroscope to produce attitude estimates during the entire range of the observed animal's body movements. Moreover, the proposed observer allows estimating the bias in gyroscope which is used to correct the angular velocity measurements in the attitude estimation step. Since, biologists use an index of DBA for evaluating the energy consumption of the moving animal; the resulting estimations are then used to extract the Dynamic Body Acceleration (DBA) of the animal. Note that, this work is necessary in Bio-logging science and allows monitoring aspects of animal's biology (behavior, movement, and physiology) and environments. The performance of the algorithm is theoretically proven and illustrated by an attitude estimation example. Moreover, the efficiency of the proposed approach is showed with a set of experiments through sensor measurements provided by an Inertial Measurement Unit (IMU). We have also included some comparison results with another method already applied in Bio-logging field in order to point out the improvements issued from the proposed approach.
\end{abstract}

Keywords: Attitude and orientation estimation, nonlinear observer, bio-signals analysis and interpretation, Bio-logging, Dynamic Body Acceleration (DBA), quaternion. 


\section{Introduction}

The rigid-body attitude and orientation estimation problems are highly motivated from various applications. For example, in rehabilitation and biomedical engineering $[1,2,3,4]$, the attitude is used in stroke rehabilitation exercises to record patient's movements in order to provide adequate feedback for the therapist. In human motion tracking and biomechanics $[5,6]$, the attitude serves as a tool for physicians to perform long-term monitoring of the patients and to study human movements during everyday activities. Moreover, the attitude estimation is extensively used in tracking of handheld microsurgical instrument [7].

Recently, the problem of attitude and orientation tracking has been treated in another scientific field: The Bio-logging. This latter is at the intersection of animal behavior and bioengineering and aims at obtaining new information on the natural world and providing new insights into the hidden lives of animal's species [8,9]. Bio-logging generally involves a free-ranging animal-attached device that records aspects of the animal's biology (behavior, movement, physiology) [10,11] and its environment. Thirty years ago, several tagging technologies such as satellite tracking (the Argos system) [12] and Time-Depth-Recorders (TDRs) [13] have been used to provide a basic knowledge on the function of free-ranging organisms. The recent advances in electronic miniaturization and digital information processing allowed researchers studying animal's biology with a high level of detail and across the full range of ecological scales.

The posture and orientation tracking of free-ranging animal represents one of the recent animal's biology aspects studied in Bio-logging. Indeed, some researches started to focus on this topic using low-cost sensors based on Micro Electro-Mechanical System (MEMS) technology as 3-axis accelerometer and 3-axis magnetometer. The main idea is how it is possible to extract the gravity components of the body animal. This information is exploited after to deduce the corresponding posture (attitude) and consequently the Dynamic Body Acceleration (DBA).

The authors assumed in [14] that the gravity measurements can be obtained from the running mean over a one second interval of the total acceleration, from accelerometer, during the motion of the animal. Then, the attitude is estimated by using the obtained gravity components and magnetometer's measurements. In a second step, on the basis on the difference between accelerometer's measurements and gravity's components, the DBA is extracted. Nevertheless, in our opinion, this approximation is not valid over time since it depends on other parameters as the animal's species and their movement's types.

In [15], a low-pass filter is used to extract the gravity's components (the lowest frequencies of the natural panel of animal's movements) from the acceleration readings. Based on this information, the authors can deduce the attitude. Note that, the use of this type of filter introduces, in many cases, an error about the attitude information since the gravity measurements are not accurately extracted by the filter. After that, a high-pass filter is used to extract the DBA of the animal since it represents the highest frequencies movements. The main idea in $[16,17]$ is based on the use of Iterated Least Squares Algorithm that combines measurements from accelerometer and magnetometer to estimate the attitude. In these works, the authors assume that the animal doesn't move at a large fraction of gravity (static and quasi-static situations of freeranging animals) which leads to consider that accelerometer's readings measure only the earth's gravity. The DBA estimation is not addressed in this paper. Other works as [18] use simply some formulas existing in 
navigation literature to deduce the attitude based on accelerometer and magnetometer measurements. The same assumptions of the work in [16,17], are considered also in [18].

To circumvent these problems, we propose in this paper the addition of 3-axis gyroscope measurements to the sensors already used. The use of gyroscope measurements in Bio-logging has never been done before in our knowledge. In fact, the previous works in this area are based only on 3-axis accelerometer and 3-axis magnetometer. Moreover, regarding other applications as aerial and marine vehicles, one can note that the application considered here (Bio-logging) is constrained by the lack of GPS data. The main guideline in this paper is to use a nonlinear observer that exploits MEMS sensors readings from the nine sensor channels cited above. The orientation reconstruction is based on an iterative procedure where the raw sensor data are combined with a previous estimate of the orientation to compute an update for the estimated orientation [19]. The proposed approach combines a strap-down system, based on the integral of the angular velocity, with an Iterated Least Squares Algorithm (ILSA) that uses Earth's magnetic field and gravity vector to calculate attitude measurements. These latter are then used to compensate those predicted by the gyroscope. Thanks to the knowledge of the estimated attitude, it is possible to reconstitute the DBA of the animal in order to evaluate its locomotor activities and daily diary [14] (sleeping, walking/flying, running, and hunting). Such information on the DBA is a major objective in a Bio-logging approach and provides important insights into some of the stresses faced by free-ranging animals especially the one studied in this work: the king penguin.

The paper is organized as follows: section 2 presents the problem statement related the Bio-logging concept. Section 3 describes the rigid body attitude and its kinematical model. Section 4 gives some details about the used sensors in this paper. The attitude nonlinear observer design and its stability conditions are depicted in section 5. Section 6 is devoted to simulation results that illustrate the efficiency of the observer. Section 7 is dedicated to a set of experiments and finally some conclusions are given in section 8 .

\section{Problem statement}

The concept of Bio-logging refers to the use of autonomous electronic devices to monitor something related to free-ranging animal itself and then to study its behaviour, physiology and ecology. The king penguin is one of the major model of diving birds studied in Strasbourg University thanks to the Bio-logging technology $[10,11]$. Then, knowing in details its foraging activities and understanding its energetic strategy need the development of new Bio-loggers. This generation of logger mainly contains 3-axis accelerometer, 3-axis gyroscope, and 3-axis magnetometer. The king penguin will be equipped with this kinematical logger (see Fig. 1). The prototype will then collect and store the sensor's data until the animal (king penguin) returns to a place where the tag can be recovered. After that all calculations are performed offline by extracting the measurements recorded on the memory card using a computer. Before deploying this new logger, the goal in this paper is to be able to convert this complex set of row data in relevant information: attitude and energy expenditure (DBA). The algorithms that will exploit the measurements from 3-axis accelerometer, 3-axis magnetometer and 3-axis gyroscope are the main concerns of this work.

\section{Rigid body attitude description}

There is one major question to be considered when designing an algorithm for rigid-body rotations that is what representation to use? In this study we will use quaternion representation due to its simplicity. 


\subsection{Coordinate systems of a rigid body}

The attitude estimation problem of rigid-body requires the transformation of measured and computed quantities between various frames. The attitude of a rigid-body is based on measurements from sensors attached to a rigid-body (the animal in our case (see Fig. 1(a))). Since the measurements are performed in the body frame, we describe in Fig. 1(b) the orientation of the body frame $F_{B}\left(x_{B}, y_{B}, z_{B}\right)$ with respect to the navigation frame $F_{I}\left(x_{I}, y_{I}, z_{I}\right)$ (attached to the earth: North, East, Down).

\subsection{The unit quaternion}

In this paper, we consider the unit quaternion that is defined by:

$$
q=q_{0}+q_{1} i+q_{2} j+q_{3} k=\left[\begin{array}{ll}
\cos \frac{\phi}{2} & \vec{u} \sin \frac{\phi}{2}
\end{array}\right]^{T}=\left[\begin{array}{c}
q_{0} \\
q_{\text {vect }}
\end{array}\right] \in Q
$$

with $q_{0}, q_{1}, q_{2}$ and $q_{3}$ are real numbers, $i, j$, and $k$ represent the components of the vector $\vec{u}$ (Euler axis), $\phi$ is the rotation angle and $q_{v e c t}=\left[\begin{array}{lll}q_{1} & q_{2} & q_{3}\end{array}\right]^{T}$ is the imaginary vector.

We invite the reader to refer to $[20,21]$ for more detailed description of quaternion algebra.

\subsection{The rigid body kinematic equation}

The quaternion $q \in Q$ satisfies the following rigid body kinematical differential equation [22]:

$\dot{q}=\frac{1}{2} q \otimes \omega_{q}$

where $\omega_{q}=\left[\begin{array}{ll}0 & \omega^{T}\end{array}\right]^{T}$ is the equivalent update quaternion relative to the angular velocity vector $\omega=\left[\begin{array}{lll}\omega_{x} & \omega_{y} & \omega_{z}\end{array}\right]^{T}$ of the rigid body measured by the gyroscope and expressed in the body frame $F_{B}$ and $\otimes$ denotes the quaternion product defined in (18). From (2) we can derive the following expression:

$\left[\begin{array}{c}\dot{q}_{0} \\ \dot{q}_{\text {vect }}\end{array}\right]=\frac{1}{2}\left[\begin{array}{c}-q_{\text {vect }}^{T} \\ I_{3} q_{0}+\left[q_{\text {vect }}^{\times}\right.\end{array}\right] \omega$

where $\left[q_{\text {vect }}^{\times}\right]$represents the skew-symmetric matrix. It is defined in appendix.

\section{Sensor measurement models}

The sensor configuration considered in this paper consists of 3-axis gyroscope, 3-axis accelerometer and 3 -axis magnetometer [23]. A detailed study of these sensors is given in [24,25].

\subsection{Gyroscope}

The 3-axes gyroscope measures the angular velocity $\omega_{G}=\left[\begin{array}{lll}\omega_{G x} & \omega_{G y} & \omega_{G z}\end{array}\right]^{T}$ in $F_{B}$ such as:

$\omega_{G}=\omega+b_{G}+\delta_{G}$

where $\omega=\left[\begin{array}{lll}\omega_{x} & \omega_{y} & \omega_{z}\end{array}\right]^{T}$ represents the real angular velocity vector expressed in the body frame $F_{B}$ and $\delta_{G} \in \mathfrak{R}^{3}$ is a zero-mean white Gaussian noise. $b_{G} \in \mathfrak{R}^{3}$ is a function varying slowly in time and representing a bias that corrupted the rate gyro measurements. This bias can be modelled by a Gauss-Markov process [26] as: 
$\dot{b}_{G}=-N^{-1} b_{G}+\delta_{b}$

where $N=\tau I_{3 \times 3}$ is a diagonal matrix of the time constant $\tau$ and $\delta_{b}=\left[\begin{array}{lll}\delta_{b x} & \delta_{b y} & \delta_{b z}\end{array}\right]^{T}$ is assumed to be modelled by zero-mean white Gaussian noise.

\subsection{Accelerometer}

The 3-axes accelerometer measures the specific force $f=\left[\begin{array}{lll}f_{x} & f_{y} & f_{z}\end{array}\right]^{T}$ in the body frame $F_{B}$ such as: $f=M(q)\left(a+g_{\text {vect }}\right)+\delta_{f}$

where $g_{\text {vect }}=\left[\begin{array}{lll}0 & 0 & g\end{array}\right]^{T}, \quad g=9.81 \mathrm{~m} / \mathrm{s}^{2}$ and $a=\left[\begin{array}{lll}a_{x} & a_{y} & a_{z}\end{array}\right]^{T}$ represent the gravity vector, the gravitational constant and the inertial (linear) acceleration expressed in $F_{I}$, respectively. $\delta_{f}=\left[\begin{array}{lll}\delta_{f x} & \delta_{f y} & \delta_{f z}\end{array}\right]^{T}$ is a zero-mean white Gaussian noise and $M(q)$ is the rotation matrix defined in appendix. Since a normalized quaternion is used, then $g_{\text {vect }}$ is also normalized to a unit vector such as:

$$
g_{\text {vect }}=\frac{\left[\begin{array}{lll}
0 & 0 & 9.81
\end{array}\right]}{\sqrt{9.81^{2}}}=\left[\begin{array}{lll}
0 & 0 & 1
\end{array}\right]^{T}
$$

\subsection{Magnetometer}

The 3-axes magnetometer measures the earth's magnetic field $h=\left[\begin{array}{lll}h_{x} & h_{y} & h_{z}\end{array}\right]^{T}$ in $F_{B}$ such as:

$$
h=M(q) m+\delta_{h}
$$

where $m=\left[\begin{array}{lll}\|m\| \cos (\theta) & 0 & \|m\| \sin (\theta)\end{array}\right]^{T}$ represents the magnetic field vector expressed in $F_{I}$.

Note that the theoretical model of this vector, nearest to the reality, is given in [27] and considers a magnetic field vector with a dip angle $\theta=60^{\circ}$ and density $\|m\|=0.5$ Gauss. Note that $\delta_{h} \in \mathfrak{R}^{3}$ is a zero-mean white Gaussian noise and $M(q)$ is the rotation matrix defined in appendix. In the same way as for the accelerometer, $m$ is normalized such as:

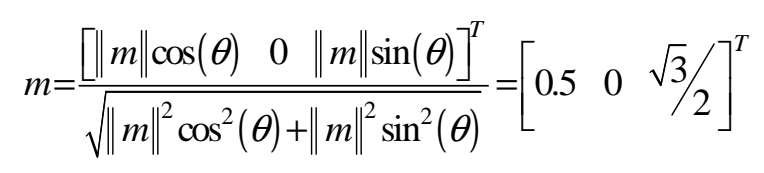

\section{Attitude nonlinear observer design}

The proposed algorithm concerns the rigid-body attitude estimation. Previous works in this area have been interested to estimate the orientation of wild animal and its DBA [14,15,16,17,18]. All these methods are based on data from two low-pass sensors as accelerometer and magnetometer and are limited to the lowest frequencies of the natural panel of animal's movements. Thus, to improve the performance of the attitude estimation in the entire range of the observed body frequencies, we propose to extend the sensor configuration by adding 3-axis gyroscope (high bandwidth). Nevertheless, this sensor is used to obtain the angular velocity but its measurements are corrupted with bias (see equations (4) and (5)). So, we suggest including the estimation of rate gyros biases in the proposed solution. 


\subsection{Design of the system dynamics}

To achieve our goal, let us consider the following nonlinear system obtained from (3) and (5):

$$
\left\{\left[\begin{array}{c}
\dot{q} \\
\dot{b_{G}}
\end{array}\right]=\left[\begin{array}{c}
\frac{1}{2}\left[\begin{array}{c}
-q_{\text {vect }}^{T} \\
I_{3} q_{0}+\left[q_{v e c t}^{\times}\right]
\end{array}\right] \omega \\
-N^{-1} b_{G}+\delta_{b} \\
y=q_{m}
\end{array}\right]\right.
$$

where $q \in \mathfrak{R}^{4}$ and $b_{G} \in \mathfrak{R}^{3}$ are the system states composed of four elements of quaternion and three elements of gyro-bias, respectively. The output of this system denoted by $q_{m} \in \mathfrak{R}^{4}$ is determined by an Aiding System (A.S) that exploits measurements from 3-axis accelerometers and 3-axis magnetometers.

Using equation (4), the nonlinear system can be written such as:

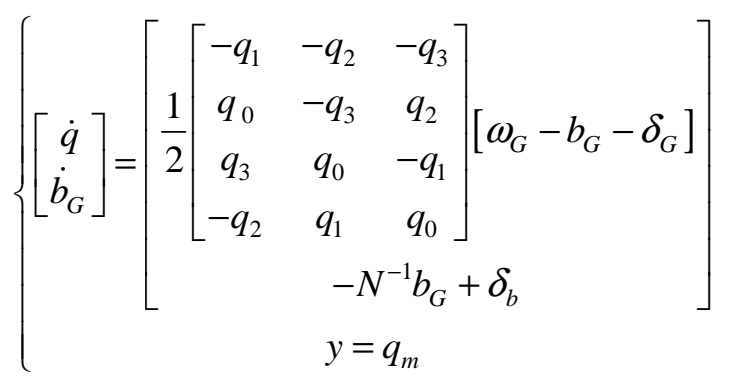

\subsection{Structure of the observation}

In order to estimate the attitude, the following nonlinear observer can be designed by using system (11):

$$
\left\{\left[\begin{array}{c}
\dot{\hat{q}} \\
\dot{\hat{b}_{G}}
\end{array}\right]=\left[\begin{array}{c}
{\left[\begin{array}{ccc}
-\hat{q}_{1} & -\hat{q}_{2} & -\hat{q}_{3} \\
\frac{1}{2} & -\hat{q}_{3} & \hat{q}_{2} \\
\hat{q}_{3} & \hat{q}_{0} & -\hat{q}_{1} \\
-\hat{q}_{2} & \hat{q}_{1} & \hat{q}_{0}
\end{array}\right]\left[\omega_{G}-\hat{b}_{G}+k_{q} q_{\text {vect }, e r}\right]}
\end{array}\right]\right.
$$

where $\hat{q}$ and $\hat{b}_{G}$ represent the estimated states. $k_{q}, k_{b} \in \Re$ represent the observer gains. $\tau$ is a time constant and $q_{\text {vect,er }}=\left[\begin{array}{lll}q_{\text {vect }, \text { er } 1} & q_{\text {vect,er } 2} & q_{\text {vect }, e r 3}\end{array}\right]^{T}$ represents the vector part of the quaternion error $q_{e r}$. This latter is obtained from the product between the measured quaternion $q_{m}$ and the estimated quaternion $\hat{q}$ :

$$
q_{e r}=\hat{q}^{-1} \otimes q_{m}=\left[\begin{array}{ll}
q_{e r 0} & q_{v e c t, e r}^{T}
\end{array}\right]^{T}
$$

Based on [28], a detailed mathematical analysis of the observer convergence and the global stability are derived [29]. Suppose that $\delta_{G}=0$ and $q_{m} \approx q$.

Theorem 1: Consider the kinematic equation (11) for a time-varying $q(t)$ and with measurements given by $q_{m}$ and $\omega_{G}$. Let $\left(\hat{q}(t), \hat{b}_{G}(t)\right)$ denote the solution of (12). Define error variables $q_{e r}=\hat{q}^{-1} \otimes q_{m}$ and $b_{e}=b_{G}-\hat{b}_{G}$. Then, the error $\left[q_{e r}^{T}(t) \quad b_{e}^{T}(t)\right]$ is globally asymptotically stable to $\left[\begin{array}{lllllll} \pm 1 & 0 & 0 & 0 & 0 & 0 & 0\end{array}\right]$. For 
almost all initial conditions $\left(q_{e r}\left(t_{0}\right), b_{e}\left(t_{0}\right)\right)$, the trajectory $\left(\hat{q}(t), \hat{b}_{G}(t)\right)$ converges to the trajectory $\left(q(t), b_{G}\right)$.

The stability theorem's proof is given in appendix.

\subsection{Measurement method of the system's output $q_{m}$}

In literature, the problem of optimal attitude determination algorithm using two sensor's measurements (vector observations) is known as the Wahba's problem [30]. A solution for this problem was provided in [16] for example to estimate the attitude of Elephant Seal. Note that this algorithm is limited to the lowest frequencies of the natural panel of animal's movements [16]. In this paper, the used algorithm provides attitude measurements $q_{m}$ as output for the system (11) and as measurements to calculate the quaternion error $q_{e r}$ for the observer. This algorithm is an estimator using the earth's magnetic field $m$ defined in (9), the gravity vector $g_{\text {vect }}$ given in (7) as the observations and the real measurements from the accelerometer and magnetometer $\left(f\right.$ and $h$ ) to deduce the attitude $q_{m}$. For that, one uses the Iterated Least Squares algorithm [16]:

\section{Iterated least squares algorithm procedure (ILSA):}

1) Take measurements of $f$ and $h$. Note that $h$ represents the magnetic field expressed in $F_{B}$.

2) Initialize $q_{m}=\left[\begin{array}{llll}1 & 0 & 0 & 0\end{array}\right]^{T}$ and $q_{e}=\left[\begin{array}{llll}1 & 0 & 0 & 0\end{array}\right]^{T}$ (quaternion error).

3) Calculate $\hat{g}_{\text {vect }}=\hat{q} \otimes f \otimes \hat{q}^{-1}$ and $\hat{m}=\hat{q} \otimes h \otimes \hat{q}^{-1}$. Note that $\hat{g}_{\text {vect }}$ and $\hat{m}$ represent the estimated gravity and magnetic fields expressed in $F_{I} \cdot \hat{q}^{-1}$ is the quaternion inverse expressed by $\hat{q}^{-1}=\left[\begin{array}{ll}\hat{q}_{0} & -\hat{q}_{v e c t}^{T}\end{array}\right]^{T}$.

4) Calculate the navigation error $\partial \hat{g}_{\text {vect }}=g_{\text {vect }}-\hat{g}_{\text {vect }}$ and do the same for $\partial \hat{m}=m-\hat{m}$ in order to form $z=\left[\begin{array}{ll}\partial \hat{m} & \partial \hat{g}_{\text {vect }}\end{array}\right]^{T}$.

5) Calculate the observation matrix: $O=\left[\left[-2[m]^{\times}\right]^{T}\left[-2\left[g_{\text {vect }}\right]^{\times}\right]^{T}\right]^{T}$

6) Calculate the pseudo-inverse $O^{*}=\left[\begin{array}{ll}O^{T} & O\end{array}\right]^{-1} O^{T}$.

7) Update the quaternion error: $q_{e}=\alpha\left[\begin{array}{ll}O^{T} & O\end{array}\right]^{-1} O^{T} z . \alpha$ is a constant that fixes the convergence speed.

8) Update the estimated quaternion: $q_{m}(k+1)=q_{m}(k) \otimes q_{e}$.

9) Return to step 3 and repeat until the convergence to a stable $q_{m}$ is reached.

\subsection{Discussion on the proposed nonlinear observer implementation}

The flow-chart of computations performed by the proposed approach is summarized in Fig. 2. A frequency analysis of the accelerometer and magnetometers signals shows that these sensors have a low bandwidth. In quasi-static motion, the acceleration and magnetic field measurements are used in the ILSA to estimate the attitude. This algorithm fails to track the accurate attitude in dynamic situations since the accelerometer measures also the DBA (considered as disturbance for the algorithm). The gyroscope's 
analysis signal shows that this sensor has a high frequency bandwidth. Its only use in dynamic situations can produce better attitude estimation but for short time due to the integration of bias $b_{G}$ (equation (4)). Then we can see that physically these three signals have complementary frequency spectra [26].

Then the resulting structure of the proposed observer in this paper is complementary: it blends low frequency region of the accelerometer and magnetometer data (based on the quaternion estimated using the ILSA), where the attitude is typically more accurate, with high frequency region (high bandwidth) of the gyroscope measurements, where the integration of the angular velocity yields better attitude estimates.

\section{Simulation results}

This section aims at illustrating the performance and accuracy of the nonlinear observer designed in (12). Some simulations were carried out using Matlab. To achieve these simulations, one starts by generating a rigid body attitude theoretical example that was the subject of angular velocity measurements over $50 \mathrm{sec}$. Then, one chooses to simulate the followings angular rate values:

$$
\begin{array}{ll}
\text { for } t \leq 25 \mathrm{sec} & \text { for } 25<t \leq 50 \mathrm{sec} \\
\left\{\begin{array}{l}
\omega_{x}(t)=-0.8 \sin (1.2 \mathrm{t}) \\
\omega_{y}(t)=1.1 \cos (0.5 \mathrm{t}) \\
\omega_{z}(t)=0.4 \sin (0.3 \mathrm{t})
\end{array}\right. & ; \quad\left\{\begin{array}{l}
\omega_{x}(t)=1.3 \sin (1.4 \mathrm{t}) \\
\omega_{y}(t)=-0.6 \cos (-0.3 \mathrm{t}) \\
\omega_{z}(t)=0.3 \sin (0.5 \mathrm{t})
\end{array}\right.
\end{array}
$$

In a first step, the kinematic differential equation (3) is used to obtain the continuous time motion in quaternion representation (see Fig. 3(a)) based on the considered angular velocity measurements in (14). This motion is used in these simulations as reference and will be estimated after by the proposed observer. In a second step, the specific force $f$ and magnetic field $h$ measurements are created using equations (6) and (8), respectively, and the rotation matrix defined in (20). The angular velocity measurements are also assumed to be corrupted by a bias. Fig. 3(b) displays the temporal evolution of this bias. Moreover, a DBA vector was added to the acceleration measurements to carry out more realistic simulations (see Fig. 3(c)). To represent the sensor imperfections, an additive random zero-mean white Gaussian noise was considered for all measurements (see Table 1). Finally, the sampling rate was chosen as $100 \mathrm{~Hz}$ for all measurements. The observer gains $k_{q}$ and $k_{b}$ that guarantee convergent estimates, are set according to the considered sensor noise levels and sampling rate as $k_{q}=100, k_{b}=200$. The constant time $\tau$ in equation (12) is chosen as $\tau=80 \mathrm{sec}$.

In this set of simulations, one chooses to initialize the actual states of the quaternion $q$ and the nonlinear observer with random values (see Table 1). Moreover, the theoretical bias and the estimated one are initialized with different initial conditions (see also Table 1). Notice that this choice allows to illustrate the convergence of the filter although it was initialized far from the actual states. It is important to stress that these simulations cannot replicate the real-world situation because the motion of the king penguin is usually unknown during its dives. Nevertheless, the numerical values are used to demonstrate the feasibility of our approach.

In order to evaluate the overall attitude and bias estimation performance, one chooses to plot the time history evolution of the estimation errors on the quaternion and bias. Fig. 4 depicts the exponential 
convergence of the estimation errors towards zero during the simulated motion. Despite the nonlinear observer and the theoretical models of the quaternion and bias were initialized with different initial conditions, one can note that the estimated quaternion and bias converge rapidly towards their theoretical values. Notice that one has tried to change the initial conditions and the same performance was obtained. These simulations demonstrated also that the nonlinear observer copes well with the rate gyro bias and the error level of the two vectors of acceleration and magnetic fields. Note that the estimated bias is used in the observer to compensate the drift in the gyroscope's measurements and consequently to increase to attitude estimation accuracy.

\section{Experimental results}

\subsection{Experimental setup}

In order to evaluate the efficiency of the proposed approach in real-world application, an experimental setup was developed resorting to an inertial and magnetic sensor module. In this study, an Inertial Measurement Units (IMU) was employed: the MTi-G, from Xsens Technologies [31], which outputs data at a rate of $100 \mathrm{~Hz}$. This MEMS device is a miniature, light weight, 3D digital output sensor (it outputs 3D accelerations, 3D angular rates, and 3D magnetic fields) with built-in bias, sensitivity, and temperature compensation. Note that the MTi-G is a GPS enhanced Attitude and Heading Reference System (AHRS). Table 2 summarizes the sensor data specifications in the MTi-G. In addition, this device is designed to track the body 3-D attitude output in Euler angles and quaternion representations. The attitude from the MTi-G is computed using an internal algorithm based on Xsens Extended Kalman Filter (XEKF) [31]. Note that the MTi-G serves as a tool for the evaluation of proposed attitude estimation algorithm efficiency, and cannot be used on free-ranging animals due to its dependence on an energy source as well as its heavy weight, makes it unsuitable for use on a large species.

\subsection{Performance evaluation}

In this section, the experiments are achieved and tested on a domestic animal such a dog to validate the proposed approach in a first step. The MTi-G is attached to the back of the animal with its $x y z$ axes aligned with those of the dog (see Fig. 5(a))). Inertial/magnetic measurements (see Fig. 5 (b)) and attitude are recorded using MTi-G during the motion of the dog and transmitted to a computer via USB port. It should be noted that the range of frequencies movements of the dog during these experiments is composed of two periods of movement with low and high frequencies respectively. In the first period (between 0 and 100sec), the motion of the dog is composed of low acceleration profile (the 3D-accelerations are between $-10 \mathrm{~m} / \mathrm{s}^{2}$ and $+10 \mathrm{~m} / \mathrm{s}^{2}$ ). In the second period (between 100sec and 170sec), the motion dynamics increase and the accelerometer outputs are between $-20 \mathrm{~m} / \mathrm{s}^{2}$ and $+20 \mathrm{~m} / \mathrm{s}^{2}$ (see Fig. 5(b)). The recorded inertial and magnetic measurements are used to generate the estimated attitude using the proposed nonlinear observer. The outputs of the MTi-G (the calculated attitude and DBA) are considered as reference of the motion of the dog. Fig. 6(a) plots the evolution of the difference between the calculated quaternion using the MTi-G and the one estimated by the proposed approach. Although some parts of the motion are with high dynamics, we 
can remark that the errors on quaternion components doesn't exceed 0.03 on $q_{0}, q_{1}, q_{2}$ and 0.05 on $q_{3}$. It is clear that this mismatch between the estimated attitude by our approach and the MTi-G is small. Then, one can conclude about the performance of the nonlinear observer in estimating the attitude of the animal even in dynamic situations. Although our approach didn't exploit a GPS data as done in MTi-G, it is able to reconstruct the orientation of the dog given by the AHRS with a small error. In our knowledge, the difference between the quaternion outputs of the Kalman filter in the MTi-G and the proposed nonlinear observer is due to the errors committed by the Xsens Kalman filter as indicated in the Technical documentation of the MTi-G (errors: pitch $/$ roll $: 1^{\circ}$ and $\left.y a w: 3^{\circ}\right)$ [31].

Dynamic Body Accelerations (DBA) of the dog can be measured using the estimated quaternion from the nonlinear observer or with the MTi-G, based on the following equation [32]:

$\hat{a}=\operatorname{inv}(M(\hat{q})) f-g_{\text {vect }}$

where $M(\hat{q})$ is defined in (20), $g_{\text {vect }}$ is the gravity vector and $f$ is the accelerometer reading.

Fig. 6(b) plots the evolution of the difference between the calculated DBA using the MTi-G and the proposed nonlinear observer. We can remark that the difference is around $0.5 \mathrm{~m} / \mathrm{s}^{2}$ on $a_{x}$ or $a_{y}$ and $0.15 \mathrm{~m} / \mathrm{s}^{2}$ on $a_{z}$ for the period between 0 and 100sec. During this time, the motion of the dog is composed of low motion dynamics (walk and trot). After that, the acceleration profile increases since the motion is faster (gallop). During this period (between 100 and 170sec), the differences on the DBA components increase (see Fig. 6(b)). In our knowledge, this difference increases due to two facts: firstly, the motion of the dog, after $100 \mathrm{sec}$, is faster than before which can cause some errors on the attitude estimation from the nonlinear observer. Secondly, it is important to stress that the Xsens Kalman Filter committed some errors on the quaternion estimation (in fast motion) [31]. These errors will be observed also on the dynamic body acceleration (using equation (15)). In conclusion, we can see that the obtained results in the Fig. 6 point out that one can estimate the DBA of the animal with a small error and satisfactory accuracy for biologists. This information will be necessary in future to assess energy expenditure of the studied animal's species especially the king penguin.

It is important to stress that the relevance of the preliminary experimental results for the final application on the penguin can be justified by two reasons. Firstly, in our knowledge, some parts of the motion dynamics of the dog will look like to those of the king penguin. Secondly, the structure of our estimation approach doesn't depend on the animal species. Consequently, the observer can estimate the motion of each animal with the same accuracy.

\subsection{Performance comparison with previous Bio-logging work}

In order to show the efficiency of the proposed approach based on the addition of gyroscope measurements, we choose to compare it with another method developed recently in the Bio-logging field [14] (it is called after in this section "method_[14]") and which uses only accelerometer and magnetometer measurements. Then, we consider the same motion of the dog and the obtained measurements showed in Fig. 5(b) are used also in the method_[14] to provide the estimated attitude. Fig. 7 plots the differences between 
the calculated Euler angles obtained from MTi-G and those estimated from the nonlinear observer and the method_[14]. Notice that the estimated Euler angles ( $\hat{\phi}:$ Roll, $\hat{\theta}:$ Pitch, $\hat{\psi}:$ Yaw) from the nonlinear observer are deduced from the estimated quaternion values based on the mathematical transformation given in [33]. We can remark from Fig. 7 that the method_[14] fails to estimate the attitude during the two periods of motion of the dog (between [0-100sec] and [100-170sec]). The difference between the MTi-G and the method_[14] reaches $50^{\circ}$ for roll, $40^{\circ}$ for pitch and $300^{\circ}$ for yaw. These degraded performances can be explained by the fact that the attitude estimation based on accelerometer and magnetometer is valid only at static and quasi-static situations (low frequencies movements) since these sensors suffer from weak dynamics (low-pass sensors). This method is not suitable to be applied for a free motion because the accelerometer outputs contain the effect of the DBA. For example, the attitude estimation error is more important when the DBA has more high values (for example at $\mathrm{t}=50 \mathrm{sec}$ (for roll and yaw), at $\mathrm{t}=100 \mathrm{sec}$ (for roll, pitch and yaw)). Figure 7 shows also that the observer is not sensitive to the effect of the DBA. It provides the smallest difference compared with the result of the method_[14]. This mismatch is always smaller than $3^{\circ}$ for roll and pitch and $5^{\circ}$ for yaw, even during fast motions (for example at $\mathrm{t}=50 \mathrm{sec}$ and $\mathrm{t}=100 \mathrm{sec}$ ). In static and quasi-static situations the mismatch is smaller than $2^{\circ}$. The conclusion is that the addition of gyroscope measurements to the accelerometer and magnetometer readings, which are used in the method_[14], can enhance the precision of attitude estimates mainly during the dynamic situations of the dog (running, fast rotation...).

In these experiments, the calculated attitude by the MTi-G is considered as reference since it uses moreover a GPS data to improve the attitude estimates quality. Then to evaluate the accuracy of the observer and the method_[14], one uses the Root Mean Square Deviation ( $R M S D$ ). Indeed, this criterion allows measuring differences between Euler angles calculated by the MTi-G and those estimated from the observer and the method_[14]. One chooses to use the following criterion with a sliding window:

$R \operatorname{MSD}_{\text {sliding }}(k)=\sqrt{\frac{\sum_{i=k}^{n+k}\left(x_{i}-\hat{x}_{i}\right)^{2}}{l}}$

where

$x_{i}$ : Euler angles (roll, pitch or yaw) calculated by the MTi-G.

$\hat{x}_{i}$ : Euler angles (roll, pitch or yaw) estimated from the chosen method (method_[14] or nonlinear observer).

$l$ : The measurement's window.

The measurement's window is chosen as $l=2$ to have a more precise evaluation of the $R M S D$ for each method. Table 3 illustrates the mean of $R M S D_{\text {sliding }}$ on roll, pitch and yaw for the two approaches. The smallest values are obtained with the nonlinear observer which prove its performance. Then, one concludes about the usefulness of gyroscope measurements to improve the quality of the motion tracking.

After that, the same the estimated attitude, given in Euler angles by the method_[14], is transformed to a quaternion representation [33] to be used in equations (20) and (15) to deduce the DBA of the dog during its 
motion. Since the components of the DBA are obtained, one calculates the norm of this acceleration using the following equation:

$\|a\|_{2}=\sqrt{a_{x}^{2}+a_{y}^{2}+a_{z}^{2}}$

The obtained values of DBA from Fig. 6(b) are used in equation (17) to calculate the corresponding norm. Fig. 8 plots the difference between the norm of DBA calculated by the MTi-G and the one estimated from each approach. In this figure, the difference between the MTi-G and method_[14] is usually high and especially at $\mathrm{t}=50 \mathrm{sec}, \mathrm{t}=80 \mathrm{sec}$, and $\mathrm{t}=100 \mathrm{sec}$ for example. This is due to the attitude estimation errors observed in Fig. 7. It is clear that the nonlinear observer is able to calculate accurately the norm of the DBA since the difference in Fig. 8 is too small even in dynamic situation of the motion's dog (between 100 and 170sec). The norm of the DBA is well estimated because the attitude estimation in Fig. 7 is too precise. In conclusion, Fig. 8 shows that the nonlinear observer provides the smallest difference compared with the result of the method_[14].

The same criterion presented in equation (16) is used to evaluate the accuracy of each method in the determination of DBA. In this equation, $x_{i}$ and $\hat{x}_{i}$ represent:

$x_{i}$ : The DBA norm calculated by the MTi-G.

$\hat{x}_{i}$ : The DBA norm estimated from the chosen method (method_[14] or nonlinear observer).

Table 3 presents also the mean of $R M S D_{\text {sliding }}$ on the norm of DBA for the nonlinear observer and the method_[14]. The smallest value is obtained also with the nonlinear observer which proves its efficiency.

\subsection{Discussion}

The estimation results obtained in the last experiments allow making some conclusions: (1) the animal's motion is composed of low and high DBA. Then, considering only the quasi-static case is not sufficient for the attitude estimation problem. (2) The attitude estimation based on accelerometer and magnetometer is valid only at low frequencies movements since these sensors suffer from weak dynamics (low-pass sensors). Then during the motion of the dog, we can see that the estimation error for method_[14] is more important when DBA happens. (3) The gyroscope has often large bandwidth (high-pass sensor) and then its addition to the accelerometer and magnetometer can improve the precision of the attitude estimation even in the period when the animal exhibits high frequencies motion (running, prey pursuit, fast rotation...). The set of experiments performed in this paper confirmed this idea since the proposed observer gives better estimation results in dynamic situations (high frequencies motion).

\section{Conclusion}

This paper concerns especially the orientation and Dynamic Body Acceleration (DBA) estimation for free-ranging animals. An approach based on the addition of gyroscope is proposed to improve the attitude and DBA estimation. The main guideline is to use a nonlinear observer coupled with an Iterated Least Squares Algorithm (ILSA) and that exploits sensor readings from 3-axis gyroscope, 3-axis accelerometer and 3-axis magnetometer. The proposed observer allows also estimating the bias in gyroscope which is used to correct the angular velocity measurements in the attitude estimation step. Afterward, the estimated attitude is 
exploited to calculate the DBA of the animal. The performance of the nonlinear observer is theoretically illustrated by an attitude estimation example. Moreover, the efficiency of the proposed approach is demonstrated with a set of experiments performed on a domestic animal under several motions. Some comparisons with another method developed previously in Bio-logging are also included to show the contribution of the proposed approach in the paper. Our future works will focus on the experimental evaluation of the algorithm using the first prototype "P3D+" that is under development in our laboratory. This logger will be attached to the king penguin and deployed in the Crozet island (South Pole). This step will give us the opportunity to know the spatial orientation and the body acceleration of the king penguin during its dives which helps the biologist to study some aspects of its behaviour and energetic index. Also, we will interest in other research to estimate the 3D position of the animal using a fusion between inertial sensors and GPS measurements to study the diving profile of the king penguin.

\section{Appendix}

\section{- Quaternion proprieties}

Let us define the quaternion product of $q_{a}=\left[\begin{array}{ll}q_{a 0} & q_{v e c t, a}^{T}\end{array}\right]^{T}$ and $q_{b}=\left[\begin{array}{ll}q_{b 0} & q_{v e c t, b}^{T}\end{array}\right]^{T}$ as:

$q_{a} \otimes q_{b}=\left[\begin{array}{cc}q_{a 0} & -q_{\text {vect }, a}^{T} \\ q_{v e c t, a} & I_{3 \times 3} q_{a 0}+\left[q_{v e c t, a}^{\times}\right.\end{array}\right]\left[\begin{array}{c}q_{b 0} \\ q_{v e c t, b}\end{array}\right]$

where $\left[q_{v e c t, a}^{\times}\right]$is the skew-symmetric matrix, defined as:

$\left[q_{\text {vect }, a}^{\times}\right]=\left[\begin{array}{c}q_{a 1} \\ q_{a 2} \\ q_{a 3}\end{array}\right]^{\times}=\left[\begin{array}{ccc}0 & -q_{a 3} & q_{a 2} \\ q_{a 3} & 0 & -q_{a 1} \\ -q_{a 2} & q_{a 1} & 0\end{array}\right]$

The rotation matrix, in terms of quaternion, can be expressed such as:

$$
M(q)=\left[\begin{array}{lll}
2\left(q_{0}^{2}+q_{1}^{2}\right)-1 & 2\left(q_{1} q_{2}+q_{0} q_{3}\right) & 2\left(q_{1} q_{3}-q_{0} q_{2}\right) \\
2\left(q_{1} q_{2}-q_{0} q_{3}\right) & 2\left(q_{0}^{2}+q_{2}^{2}\right)-1 & 2\left(q_{0} q_{1}+q_{2} q_{3}\right) \\
2\left(q_{0} q_{2}+q_{1} q_{3}\right) & 2\left(q_{2} q_{3}-q_{0} q_{1}\right) & 2\left(q_{0}^{2}+q_{3}^{2}\right)-1
\end{array}\right]
$$

- Proof of the stability theorem

Proof: Let us consider the two error equations given by:

$$
\begin{aligned}
& q_{e r}=\left[\begin{array}{llll}
q_{e r 0} & q_{e r 1} & q_{e r 2} & q_{e r 3}
\end{array}\right]^{T}=\hat{q}^{-1} \otimes q_{m} \\
& b_{e}=\left[\begin{array}{lll}
b_{e x} & b_{e y} & b_{e z}
\end{array}\right]^{T}=b_{G}-\hat{b}_{G}
\end{aligned}
$$

Suppose that $q_{m} \approx q$ and using the definition of quaternion product $\otimes$ given in (18), equation (21) can be written as:

$$
q_{e r}=\left[\begin{array}{c}
q_{0} \hat{q}_{0}+q_{1} \hat{q}_{1}+q_{2} \hat{q}_{2}+q_{3} \hat{q}_{3} \\
-q_{0} \hat{q}_{1}+q_{1} \hat{q}_{0}+q_{2} \hat{q}_{3}-q_{3} \hat{q}_{2} \\
-q_{0} \hat{q}_{2}-q_{1} \hat{q}_{3}+q_{2} \hat{q}_{0}+q_{3} \hat{q}_{1} \\
-q_{0} \hat{q}_{3}+q_{1} \hat{q}_{2}-q_{2} \hat{q}_{1}+q_{3} \hat{q}_{0}
\end{array}\right]
$$


Now, differentiating (22) and (23), then one obtains:

$\left\{\begin{array}{l}\dot{q}_{e r}=\left[\begin{array}{l}\dot{q}_{0} \hat{q}_{0}+q_{0} \dot{\hat{q}}_{0}+\dot{q}_{1} \hat{q}_{1}+q_{1} \dot{\hat{q}}_{1}+\dot{q}_{2} \hat{q}_{2}+q_{2} \dot{\hat{q}}_{2}+\dot{q}_{3} \hat{q}_{3}+q_{3} \dot{\hat{q}}_{3} \\ -\dot{q}_{0} \hat{q}_{1}-q_{0} \dot{\hat{q}}_{1}+\dot{q}_{1} \hat{q}_{0}+q_{1} \dot{\hat{q}}_{0}+\dot{q}_{2} \hat{q}_{3}+q_{2} \dot{\hat{q}}_{3}-\dot{q}_{3} \hat{q}_{2}-q_{3} \dot{\hat{q}}_{2} \\ -\dot{q}_{0} \hat{q}_{2}-q_{0} \dot{\hat{q}}_{2}-\dot{q}_{1} \hat{q}_{3}-q_{1} \dot{\hat{q}}_{3}+\dot{q}_{2} \hat{q}_{0}+q_{2} \dot{\hat{q}}_{0}+\dot{q}_{3} \hat{q}_{1}+q_{3} \dot{\hat{q}}_{1} \\ -\dot{q}_{0} \hat{q}_{3}-q_{0} \dot{\hat{q}}_{3}+\dot{q}_{1} \hat{q}_{2}+q_{1} \dot{\hat{q}}_{2}-\dot{q}_{2} \hat{q}_{1}-q_{2} \dot{\hat{q}}_{1}+\dot{q}_{3} \hat{q}_{0}+q_{3} \dot{\hat{q}}_{0}\end{array}\right] \\ \dot{b}_{e}=\dot{b}_{G}-\dot{\hat{b}}_{G}\end{array}\right.$

Substitute $\quad \dot{q}=\left[\begin{array}{llll}\dot{q}_{0} & \dot{q}_{1} & \dot{q}_{2} & \dot{q}_{3}\end{array}\right]^{T}, \quad \dot{b}_{G}=\left[\begin{array}{lll}\dot{b}_{G x} & \dot{b}_{G y} & \dot{b}_{G z}\end{array}\right]^{T}, \dot{\hat{q}}=\left[\begin{array}{llll}\dot{\hat{q}}_{0} & \dot{\hat{q}}_{1} & \dot{\hat{q}}_{2} & \dot{\hat{q}}_{3}\end{array}\right]^{T}$ and $\quad \dot{\hat{b}}_{G}=\left[\begin{array}{lll}\dot{\hat{b}}_{G x} & \dot{\hat{b}}_{G y} & \dot{\hat{b}}_{G z}\end{array}\right]^{T}$ given in (11) and (12).

Finally, the observation error dynamic's is written as follows:

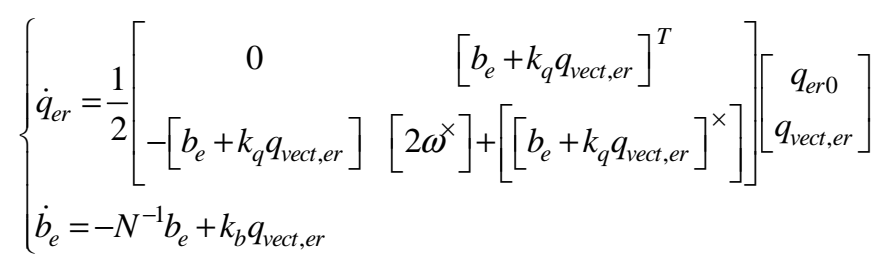

It is easily verified that:

$\left[\begin{array}{ll}q_{e r}^{T} & b_{e}^{T}\end{array}\right]=\left[\begin{array}{lllllll}+1 & 0 & 0 & 0 & 0 & 0 & 0\end{array}\right]$

and

$\left[\begin{array}{ll}q_{e r}^{T} & b_{e}^{T}\end{array}\right]=\left[\begin{array}{lllllll}-1 & 0 & 0 & 0 & 0 & 0 & 0\end{array}\right]$

are the equilibrium states of the error dynamics (25).

Let us consider a domain $D$ as:

$D=\left\{q_{e r} \in \mathfrak{R}^{4 \times 1} /-1 \leq q_{e r}(i) \leq 1,\{\mathrm{i}=1 . .4\}\right.$ and $\left.b_{e} \in \mathfrak{R}^{3 \times 1} /-\infty<b_{e}(i)<+\infty,\{\mathrm{i}=1 . .3\}\right\}$

Let us define two candidate Lyapunov functions $V_{1}$ and $V_{2}$. These functions are continuous, positive definite, bounded and belong to the class $C^{2}$ as:

$V_{1}=\frac{1}{2} b_{e}^{T} b_{e}+k_{b}\left(\left(1-q_{e r 0}\right)^{2}+q_{\text {vect }, e r}^{T} q_{\text {vect }, e r}\right)$, if $q_{e r 0} \geq 0$

$V_{2}=\frac{1}{2} b_{e}^{T} b_{e}+k_{b}\left(\left(1+q_{e r 0}\right)^{2}+q_{v e c t, e r}^{T} q_{v e c t, e r}\right)$, if $q_{e r 0}<0$

In our case, the motion can change randomly, and then $q_{e r 0}$ can take positive or negative value according to the value of $\phi$. If we choose the case $q_{e r 0} \geq 0$, then we consider the Lyapunov function in (29). Equation (29) can be written also as:

$V=\frac{1}{2} b_{e}^{T} b_{e}+k_{b}\left(2\left(1-q_{e r 0}\right)\right)$

When differentiating (31) and using (25), one obtains:

$\dot{V}=-2 k_{b} \dot{q}_{e r 0}+b_{e}^{T} \dot{b}_{e}$

Finally, one obtains:

$\dot{V}=-k_{b} k_{q} q_{v e c t, e r}^{T} q_{v e c t, e r}-b_{e}^{T} N^{-1} b_{e}$ 
Since $0 \leq q_{e r 0} \leq 1$, then:

$q_{\text {vect }, \text { er }}^{T} q_{\text {vect }, \text { er }}=1-q_{\text {er } 0}^{2} \geq 0$

When the gains $k_{q}, k_{b}$ are positive constants, we can write:

$\dot{V} \leq 0$

It is clear that $\dot{V}$ is negative semi-definite and for equilibrium states (26) and (27), the condition $V\left(q_{e r}, b_{e}\right)=\dot{V}\left(q_{e r}, b_{e}\right)=0$ is satisfied. Thus, $\left(q_{v e c t, e r}, b_{e}\right) \rightarrow 0$ and consequently $q_{e r 0} \rightarrow \pm 1$ (the norm of $q_{e r}$ is always equal 1). In the same way, for the case $q_{e r 0}<0$, the associated Lyapunov function (30) leads to the same result given by (33).

Let $\Upsilon=\left\{\left(q_{e r}, b_{e}\right) \in D / \dot{V}\left(q_{e r}, b_{e}\right)=0\right\}$. Therefore, the only solution that can stay identically in $\Upsilon$ is the trivial solutions in (26) and (27). Now, applying Krasovskii-LaSalle's principle [34], one can conclude that the equilibrium states (26) and (27) are globally asymptotically stable, which ends the proof.

\section{Acknowledgements}

The authors would like to thank both of the Alsace and Champagne-Ardenne regions within the framework of the project (NaviMeles) for their financial support and PhD students of AUTO team (CReSTIC) especially Mr. T. Bouarar and Miss. D. Jabri for their helps during experiments on the dog.

\section{References}

[1] H. Zhou, H. Hu, N. D. Harris, J. Hammerton, Applications of wearable inertial sensors in estimation of upper limb movements, Biomedical Signal Processing and control 1 (January (1)) (2006) 22-32.

[2] H. Zhou, H. Hu, Human motion tracking for rehabilitation-A survey, Biomedical Signal Processing and control 3 (January (1)) (2008) 1-18.

[3] R. Zhu, Z. Zhou, A real-time articulated human motion tracking using tri-axis inertial/magnetic sensors package, IEEE Transactions on Neural Systems and Rehabilitation Engineering, 12 (June(2)) (2004) 295-302.

[4] A. M. Sabatini, Quaternion-based Extended Kalman Filter for determining orientation by inertial and magnetic sensing, IEEE Transactions on Biomedical Engineering 53 (July (7)) (2006) 1346-1356.

[5] A. Gallagher, Y. Matsuoka, W. T. Ang, An efficient real-time human posture tracking algorithm using low-cost inertial and magnetic sensors, IEEE/RSJ International Conference on Intelligent Robots and Systems, Sendai, Japan, 2004, pp. 2967-2972.

[6] K. J. O’Donovan, R. Kamnik, D. T. O'Keeffe, G. M. Lyons, A inertial and magnetic sensor based technique for joint angle measurement, Journal of Biomechanics 40 (12) (2007) 2604-2611.

[7] W. T. Ang, P. K. Khosla, C. N. Riviere, Kalman filtering for real-time orientation tracking of handheld microsurgical instrument, IEEE/RSJ International Conference on Intelligent Robots and Systems, Sendai, Japan, 2004, pp. 2574-2580.

[8] C. Rutz, G. C. Hays, New frontiers in biologging science, Biology letters, (March) (2009).

[9] Y. Ropert-Coudert, M. Beaulieu, N. Hanuise, A. Kato, Diving into the world of biologging, Endangered Species Research, (April) (2009). 
[10] L. G. Halsey, Y. Handrich, A. Fahlman, A. Schmidt, C. A. Bost, R. L. Holder, A. J. Woakes, P. J. Butler, Finescale analyses of diving energetics in king penguins Aptenodytes patagonicus: how behaviour affect costs of a foraging dive, Marine Ecology Progress Series 344 (2007).

[11] C. A. Bost, Y. Handrich, P. J. Butler, A. Fahlman, L. G. Halsey, A. J. Woakes, Y. Ropert-Coudert, Change in dive profiles as an indicator of feeding success in king and Adélie penguins, Deep-Sea Research II 54 (3-4) (2007) 248255.

[12]B. J. Le Boeuf, D. E. Crocker, D. P. Costa, S. P. Blackwell, P. M. Webb, D. S. Houser, Foraging ecology of northern elephant seals, Ecological Monographs 70 (3) (2000) 353-382.

[13] G. L. Kooyman, Genesis and evolution of bio-logging devices: 1963-2002, Memoirs of the National Institute of Polar Research 58 (2004) 148-154.

[14] R. Wilson, E. L. C. Shepard, N. Liebsch, Prying into the intimate details of animal lives: use of a daily diary on animals, Endangered Species Research 4 (January) (2008) 123-137.

[15] S. Watanabe, M. Isawa, A. Kato, Y. Coudert, Y. Naito, A new technique for monitoring the behaviour of terrestrial animals; a case study with the domestic cat, Applied Animal Behaviour Science 94 (2005) 117-131.

[16] G. H. Elkaim, E. B. Decker, G. Oliver, B. Wright, Marine Mammal Marker (MAMMARK) dead reckoning sensor for In-Situ environmental monitoring, IEEE Position, Location and Navigation Symposium, Monterey, USA, April 2006, pp. 976-987.

[17] G. H. Elkaim, E. B. Decker, G. Oliver, B. Wright, Initial results from an In Situ Environmental Monitoring Marine Mammal Tag, IEEE Position, Location and Navigation Symposium, May 2008, pp. 912-922.

[18] M. P. Johnson, P. L. Tyack, A digital acoustic recording tag for measuring the response of wild marine mammals to sound, IEEE Journal of Oceanic Engineering 28 (January (1)) (2003) 3-12.

[19] H. Fourati, N. Manamanni, L. Afilal, Y. Handrich, Rigid body motions estimation using inertial sensors: Biologging application, $7^{\text {th }}$ IFAC Symposium on Modelling and Control in Biomedical Systems (including Biological Systems), Alborg, Danemark, August 2009.

[20] J. H. Conway, D. A. Smith, On quaternions and octonions: Their geometry, arithmetic and symmetry, A K Peters. Ltd, Massachusetts, 2003.

[21] J. B. Kuipers, Quaternion and Rotation sequences, Princeton, NJ: Princeton University Press, 1999.

[22] M. D. Shuster, A survey of attitude representations, Journal of the Astronautical Science 41 (October-December (4)) (1993) 493-517.

[23]H. Fourati, N. Manamanni, L. Afilal, Y. Handrich, Nonlinear attitude estimation based on fusion of inertial and magnetic sensors: Bio-logging application, $2^{\text {nd }}$ IFAC International Conference on intelligent Control Systems and Signal Processing, Istanbul, Turkey, September 2009, .

[24]M. Bao, Analysis and design principles of MEMS devices, Elseiver B. V., 2007.

[25] S. Beeby, G. Ensell, M. Kraft, N. White. MEMS Mechanical Sensors, Artech House House Publishers, 2004.

[26]R. G. Brown, P. Y. C. Hwang, Introduction to Random Signal and Applied Kalman Filtering. $3^{\text {rd }}$ Ed. New York: John Wiley, 1997.

[27] Astrosurf, Available: http://www.astrosurf.com/luxorion/terre-champ-magnetique2.htm (September) (2009).

[28] S. Salcudean, S, A globally convergent angular velocity observer for rigid body motion, IEEE Transactions on Automatic Control 36 (12) (1991) 1493-1497.

[29] H. Fourati, N. Manamanni, L. Afilal, Y. Handrich, A complementary observer-based approach for the estimation of motion in rigid bodies using inertial and magnetic sensors, IEEE Multi-Conference on Systems and ControlConference on Control Applications (MSC-CCA), Yokohama, Japan, September 2010. 
[30] G. Wahba, G. A least squares estimate of spacecraft attitude, SIAM, Review 7 (3) (1965) 409.

[31] Xsens Technologies, Available: http://www.xsens.com (September) (2009).

[32] H. Fourati, N. Manamanni, L. Afilal, Y. Handrich, A rigid body attitude estimation for Bio-logging application: A quaternion-based nonlinear filter approach, IEEE/RSJ International Conference on Intelligent Robots and Systems (IROS), St. Louis, USA, October 2009, pp.558-563.

[33] W. F. Phillips, C. E. Hailey, G. A. Gebert, Review of attitude representations used for aircraft kinematics, Journal of Aircraft 38 (July-August (4)) 718-223.

[34]H. Khalil, Nonlinear Systems, $3^{\text {rd }}$ ed. NJ: Prentice-Hall, 2002. 


\section{$\underline{\text { Tables }}$}

Table 1

Noise characteristics in the used sensors and initial conditions for the desired states and the nonlinear observer

\begin{tabular}{|c|c|c|c|c|c|c|}
\hline Sensors & Parameters & $\begin{array}{l}\text { Standard } \\
\text { deviations }\end{array}$ & Units & & Quaternion & Bias \\
\hline Accelerometer & $\delta_{f}$ & 0.002 & $\mathrm{~m} / \mathrm{s}^{2}$ & \multirow{2}{*}{$\begin{array}{l}\text { Theoretical } \\
\text { values }\end{array}$} & \multirow{2}{*}{$q\left(t_{0}\right)=\left[\begin{array}{llll}1 & 0 & 0 & 0\end{array}\right]^{T}$} & \multirow{2}{*}{$b\left(t_{0}\right)=\left[\begin{array}{lll}-2 & 1 & 0.5\end{array}\right]^{T}$} \\
\hline Magnetometer & $\delta_{h}$ & 0.007 & Gauss & & & \\
\hline Gyroscope & $\delta_{G}$ & 0.01 & $\mathrm{rad} / \mathrm{s}$ & \multirow{2}{*}{$\begin{array}{l}\text { Observer } \\
\text { values }\end{array}$} & \multirow{2}{*}{$\hat{q}\left(t_{0}\right)=\left[\begin{array}{llll}0.3 & 0.5 & 0.8 & 0.7\end{array}\right]^{T}$} & \multirow{2}{*}{$\hat{b}\left(t_{0}\right)=\left[\begin{array}{lll}0 & 0 & 0\end{array}\right]^{T}$} \\
\hline Bias & $\delta_{b}$ & 0.01 & $\mathrm{rad} / \mathrm{s}$ & & & \\
\hline
\end{tabular}

Table 2

Performance specification of MTi-G

\begin{tabular}{llll}
\hline Sensor performance & Rate of turn & Acceleration & Magnetic field \\
\hline Full scale & $\pm 300 \mathrm{deg} / \mathrm{s}$ & $\pm 50 \mathrm{~m} / \mathrm{s}^{2}$ & $\pm 750 \mathrm{mGauss}$ \\
Bias stability & $1 \mathrm{deg} / \mathrm{s}$ & $0.02 \mathrm{~m} / \mathrm{s}^{2}$ & $0.1 \mathrm{mGauss}$ \\
Noise & $0.05 \mathrm{deg} / \mathrm{s} / \sqrt{\mathrm{Hz}}$ & $0.002 \mathrm{~m} / \mathrm{s}^{2} / \sqrt{\mathrm{Hz}}$ & $0.5 \mathrm{mGauss}$ \\
Alignment error & $0.1 \mathrm{deg}$ & $0.1 \mathrm{deg}$ & $0.1 \mathrm{deg}$ \\
\hline
\end{tabular}

Table 3

Mean of $R M S D_{\text {sliding }}$ on Euler angles and DBA for the nonlinear observer and the method_[14]

\begin{tabular}{lll}
\hline Methods & Nonlinear observer & Method_[14] \\
\hline Mean of $R M S D_{\text {sliding }}$ (Roll) & 0.4887 & 3.5164 \\
Mean of $R M S D_{\text {sliding }}$ (Pitch) & 0.3813 & 3.5464 \\
Mean of $R M S D_{\text {sliding }}$ (Yaw) & 1.9654 & 9.5164 \\
Mean of $R M S D_{\text {sliding }}$ (DBA) & 0.0579 & 0.5162 \\
\hline
\end{tabular}




\section{$\underline{\text { List of Figures }}$}

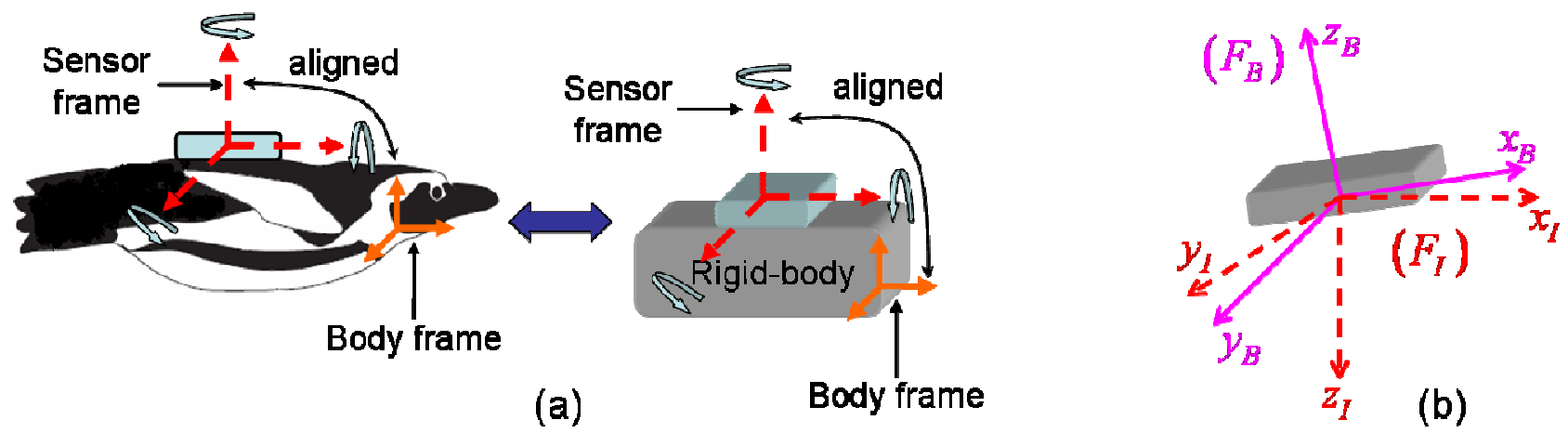

Fig. 1. (a) The schematic diagram of an electronic device (logger) attached to a king penguin - (b) The coordinate systems of a rigid body

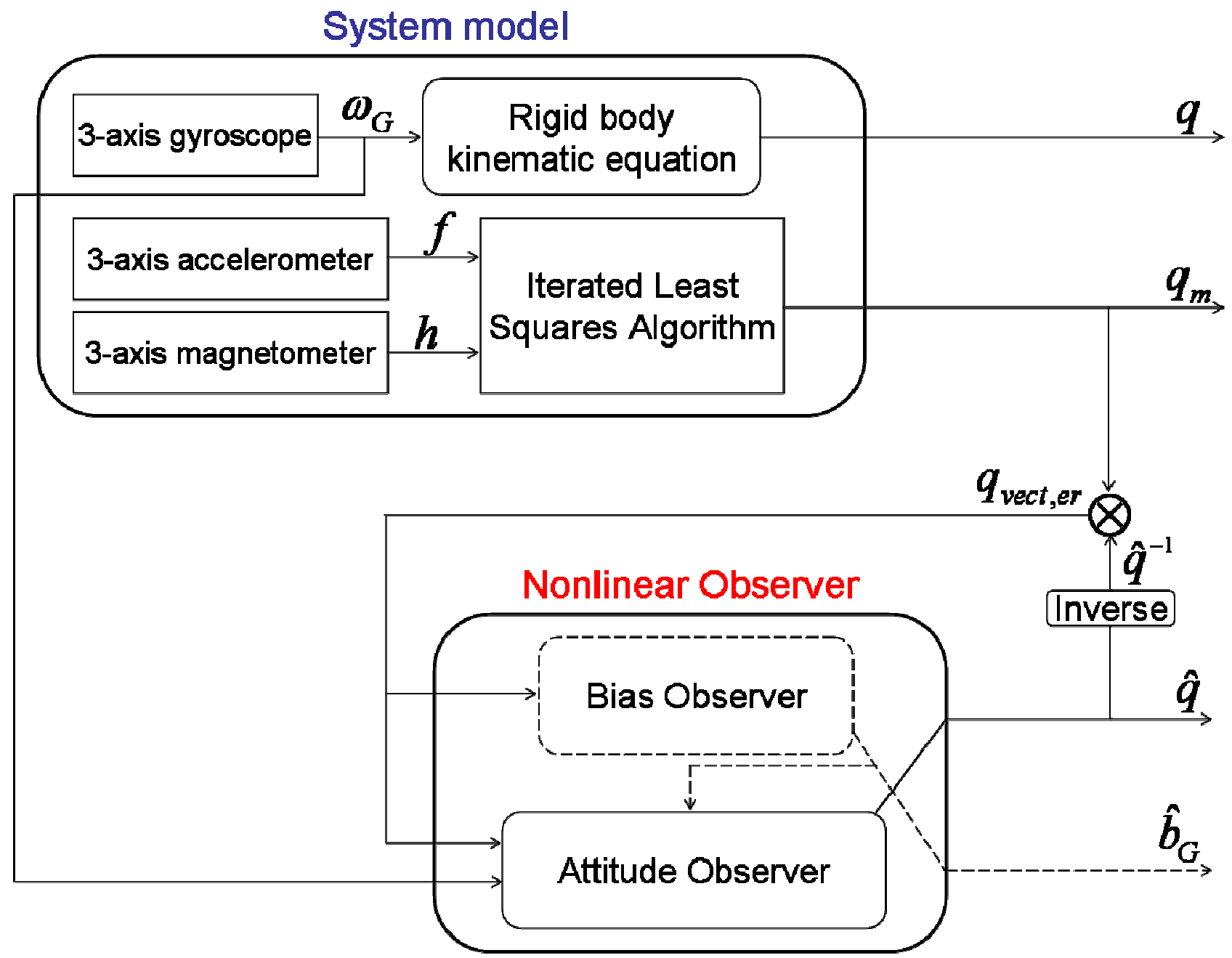

Fig. 2. Block diagram for the attitude and bias estimation algorithm 
(a)
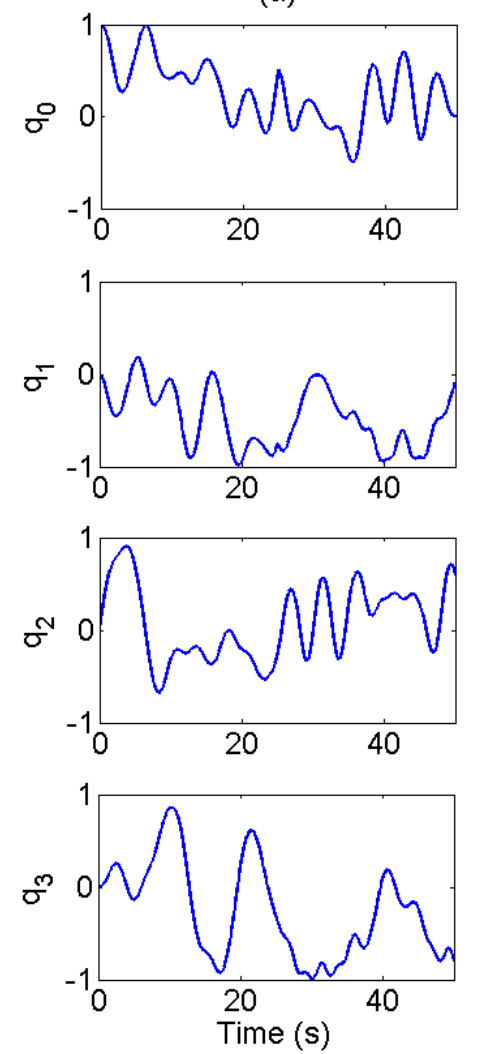

(b)
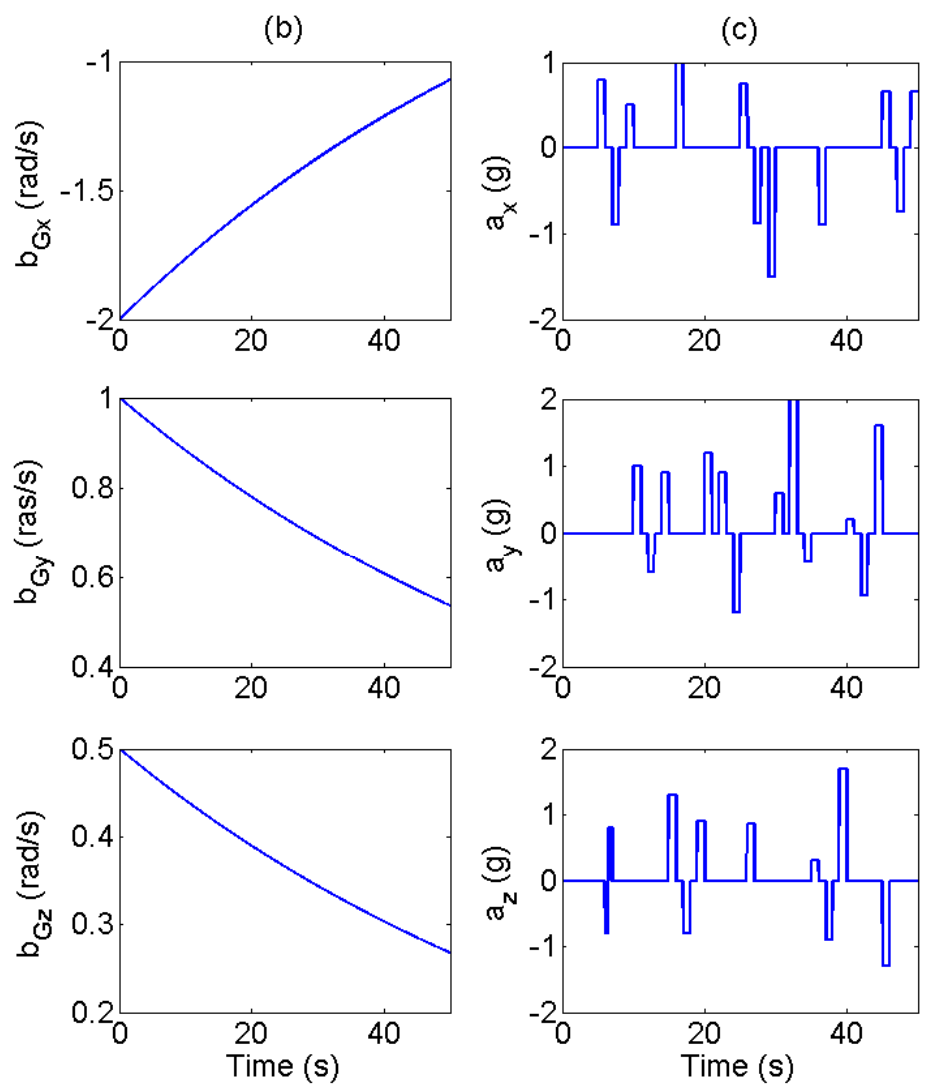

Fig. 3. Theoretical models (a) The quaternion $q=\left[\begin{array}{llll}q_{0} & q_{1} & q_{2} & q_{3}\end{array}\right]^{T}-$ (b) The bias $b_{G}=\left[\begin{array}{lll}b_{G x} & b_{G y} & b_{G z}\end{array}\right]^{T}-(\mathrm{c})$ The Dynamic Body Acceleration (DBA) $a=\left[\begin{array}{lll}a_{x} & a_{y} & a_{z}\end{array}\right]^{T}$ 
(a)
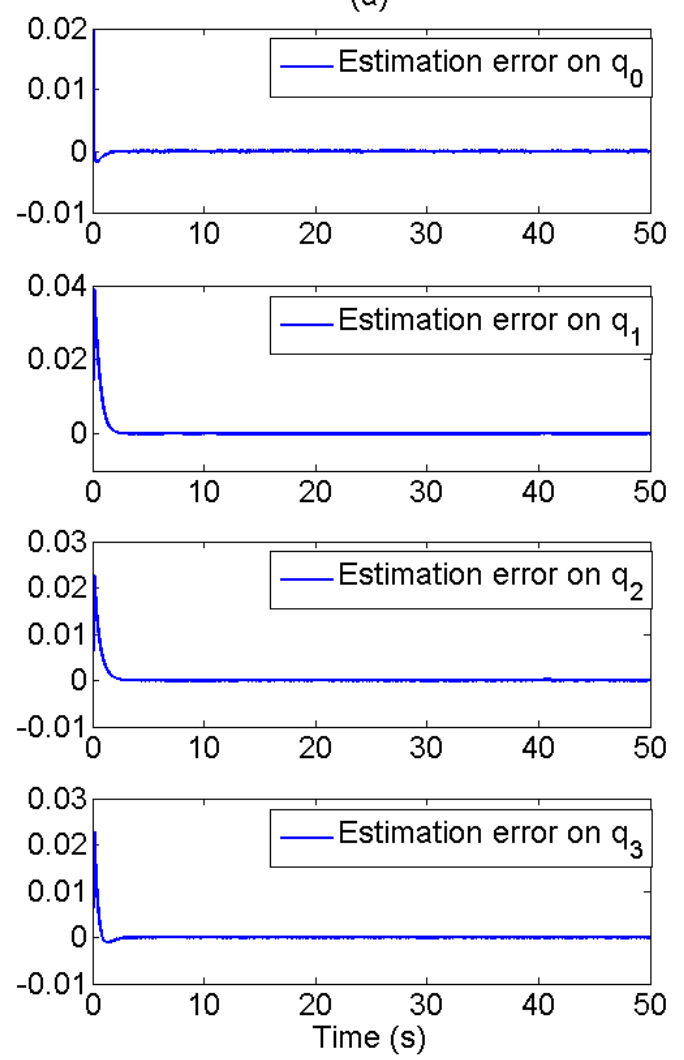

(b)
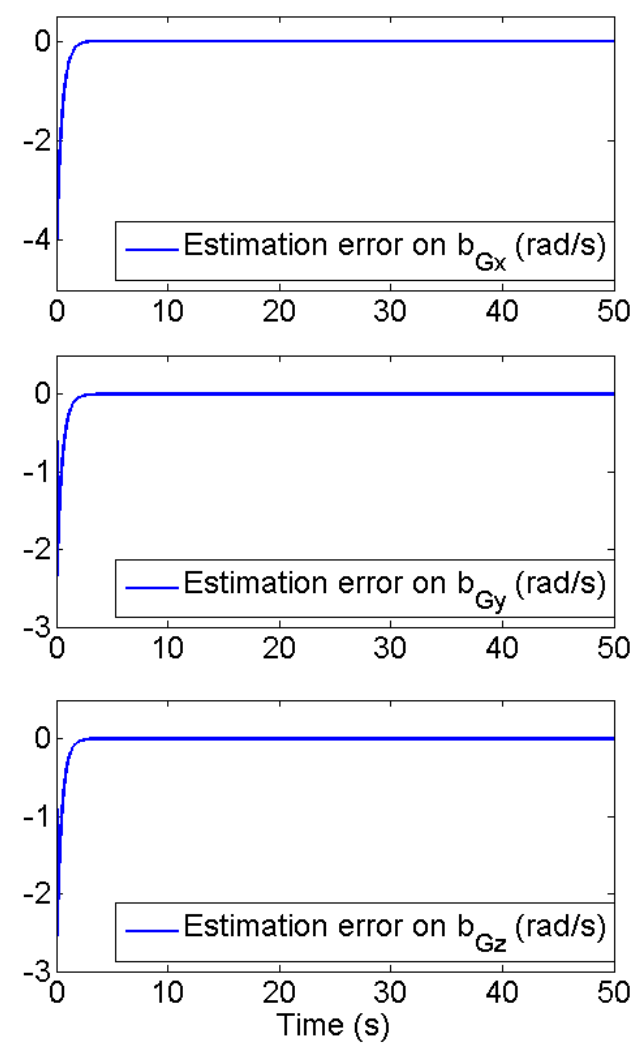

Fig. 4. Estimation errors (a) on the quaternion - (b) on the bias

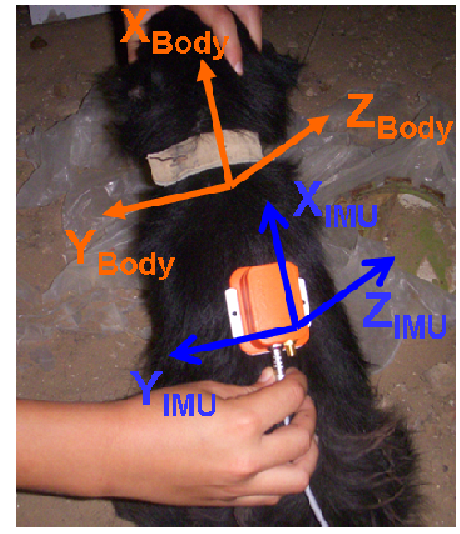

(a)
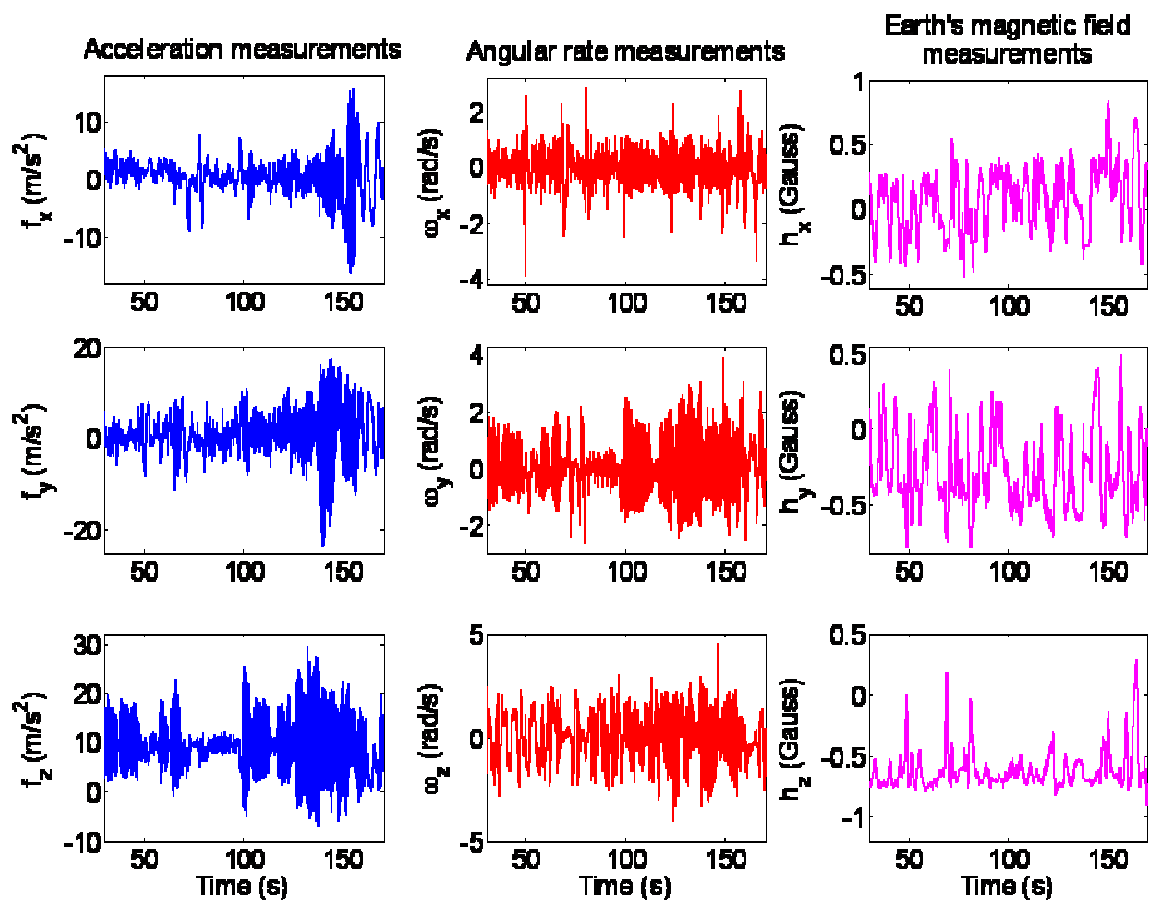

(b)

Fig. 5. (a) The MTi-G attached to the back of the dog - (b) Acceleration, angular rate and magnetic field measurements from the MTi-G during the motion of the dog 
(a)
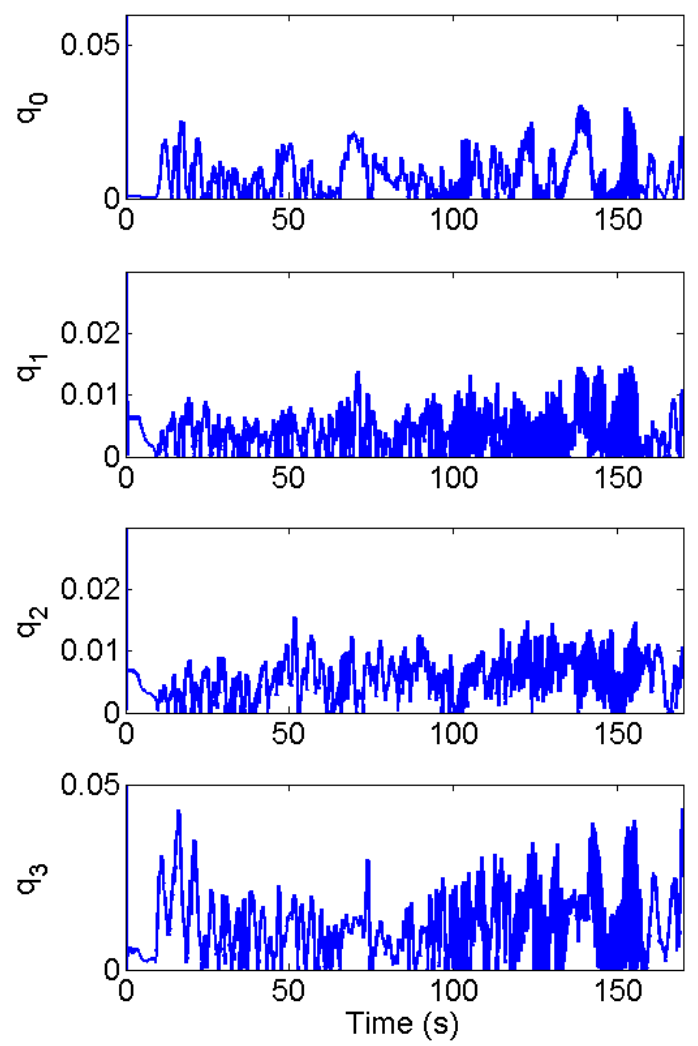

(b)
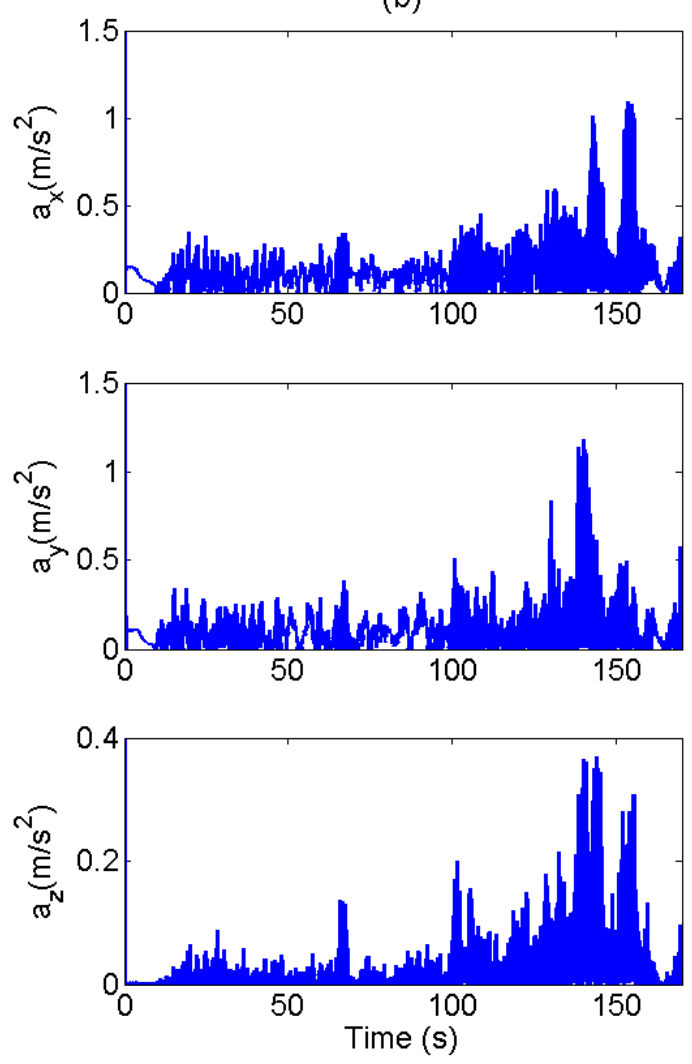

Fig. 6. (a) Difference between quaternion values calculated by the MTi-G and those estimated from the proposed observer - (b) Difference between Dynamic Body Acceleration values calculated by the MTi-G and those estimated from the proposed observer 

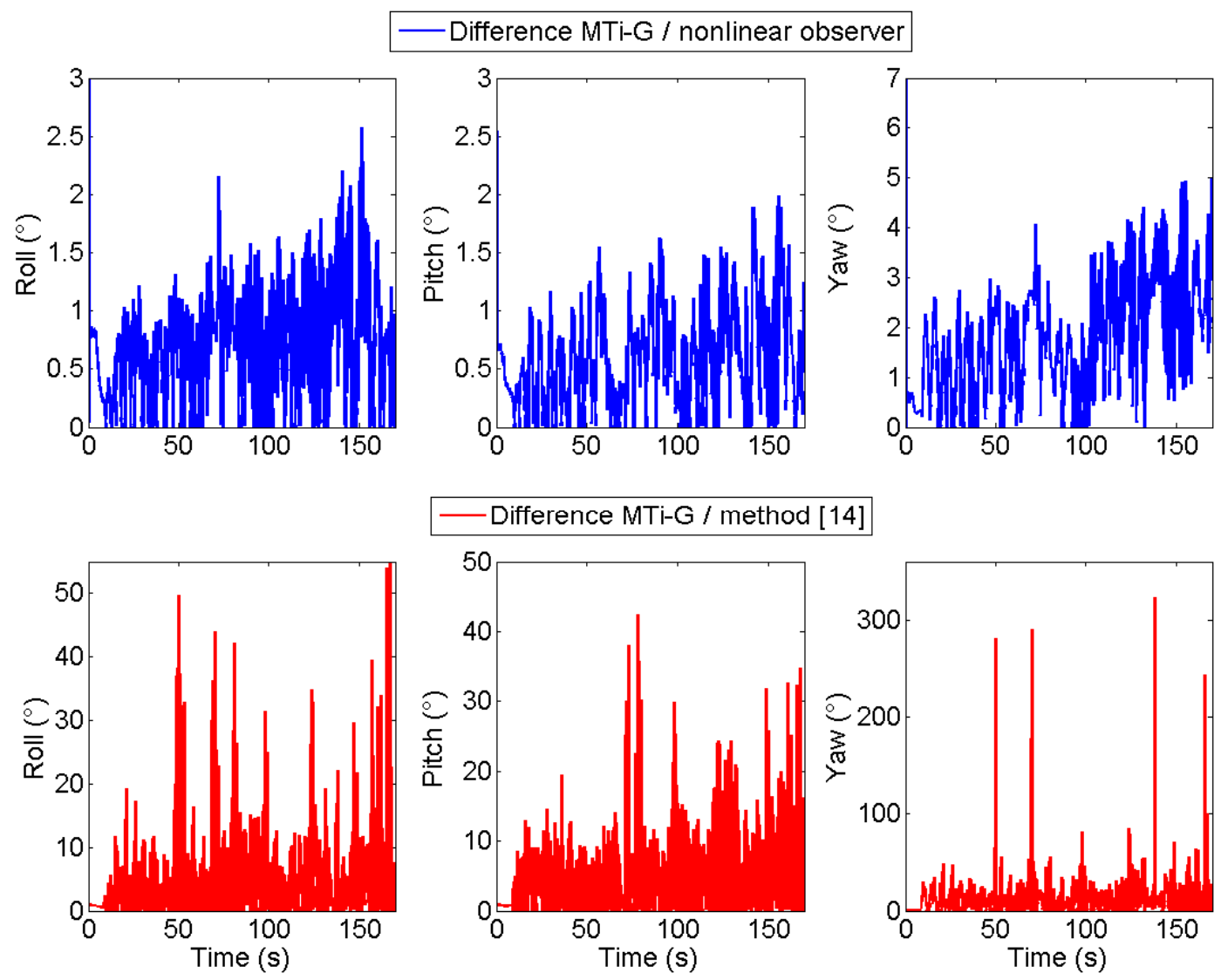

Fig. 7. Differences between Euler angles calculated by the MTi-G and those estimated from the nonlinear observer and the method_[14] 

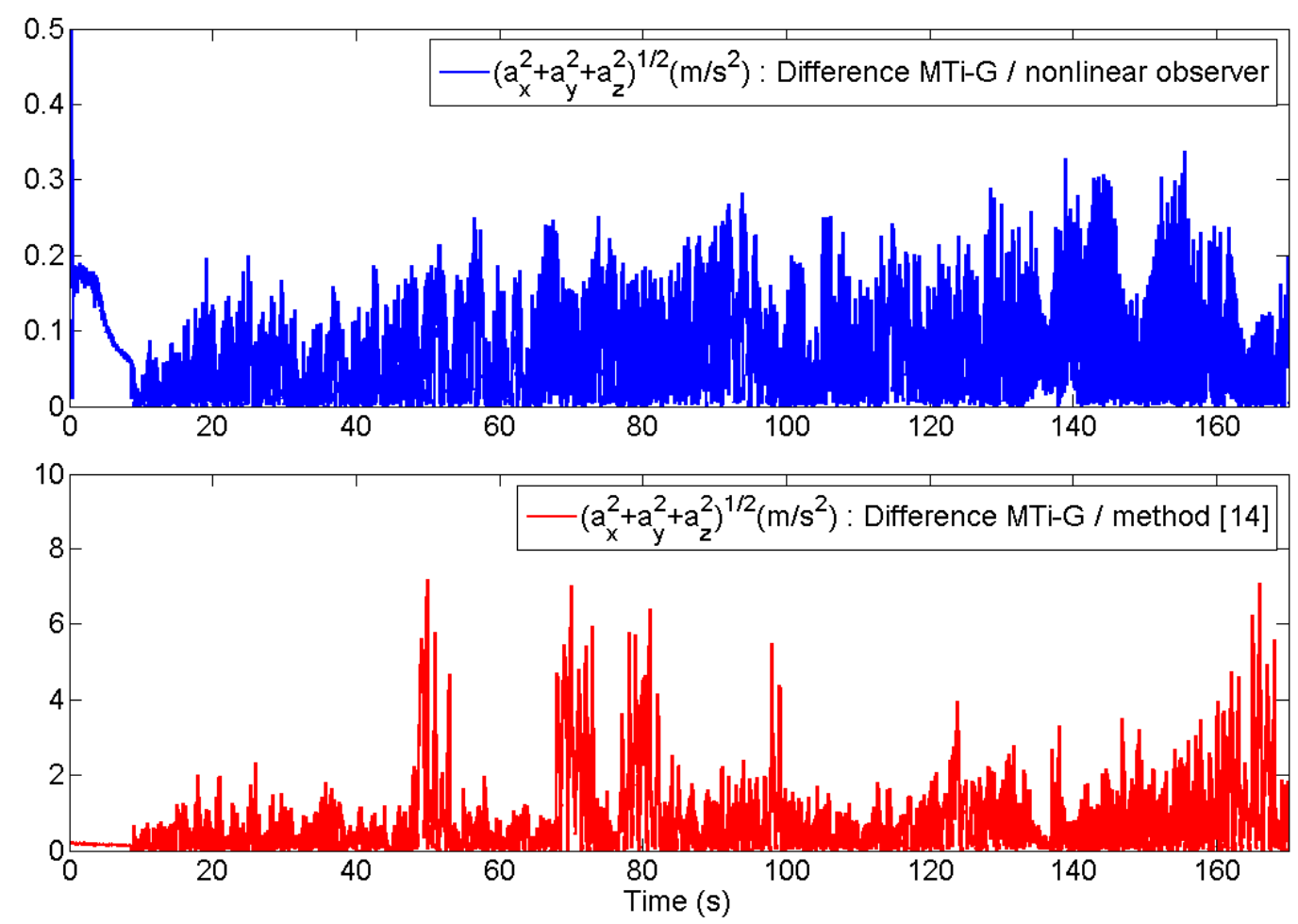

Fig. 8. Differences between the Dynamic Body Acceleration norm calculated by the MTi-G and the one estimated by the nonlinear observer and the method_[14] 